Use of Three-Dimensional Computed Tomography Reconstruction and RealTime Ultrasound-Guided Spinal Anaesthesia in a Patient with Ankylosing

\title{
Spondylitis: A Case Report
}

David MH Lam ${ }^{1 *}$, John CY Chan², Henry CY Mak', Yu Fai Chung' and Timmy CW Chan ${ }^{1}$

${ }^{1}$ Department of Anaesthesiology, Queen Mary Hospital, Hong Kong

${ }^{2}$ Department of Radiology, Queen Mary Hospital, Hong Kong

"Corresponding author: David MH Lam, Department of Anaesthesiology, F2, Main Block, Queen Mary Hospital, 102 Pok Fu Lam Road, Hong Kong SAR. Phone: +852 2255 5791, Email: davidmhlam@hotmail.com

\begin{abstract}
Background: This case report describes the use of two techniques, three-dimensional computed tomography
\end{abstract}

(3D-CT) reconstruction and real-time ultrasound (US), for performing spinal anesthesia in a patient with ankylosing spondylitis, the former for accurate pre-operative assessment of feasibility of spinal anaesthesia (SA) and the latter for aiding proper needle insertion. This is the first time this combination of techniques is used and documented.

Case Presentation: A woman with severe ankylosing spondylitis and extensive retrosternal goitre was admitted for intertrochanteric femoral fracture. Preoperative Computed Tomography (CT) and 3D-CT reconstruction of the lumbrosacral spine were used to identify unfused interlaminar spaces before SA was successfully performed under real-time US guidance for proximal femoral nail antirotation.

Conclusion: 3D-CT provides an objective way of identifying unfused interlaminar spaces to assess the feasibility of a SA while real-time US-guided SA offers the advantage of allowing manipulation of needle under direct vision without exposure to radiation.

Keywords: Three-dimensional computed tomography; 3D-CT; Real time ultrasound guided spinal anesthesia; Regional anaesthesia; Spinal anaesthesia; Ankylosing spondylitis

Abbreviations: 3D-CT: Three-Dimensional Computed Tomography; AS: Ankylosing Spondylitis; CT: Computed Tomography; SA: Spinal Anesthesia; US: Ultrasound

\section{Background}

The reported prevalence of Ankylosing Spondylitis (AS) is approximately $1 \%$ in men and $0.5 \%$ in women in Caucasians 1 and $0.42 \%$ in men and $0.15 \%$ in women in the Chinese population2. Histologically, affected joints are infiltrated by plasma cells, lymphocytes, mast cells, and macrophages; these cells are gradually replaced by fibrocartilage, resulting in rigid, kyphotic spinal deformity. Intubation is particularly challenging in these patients due to the kyphotic spinal deformity, possible atlantoaxial subluxation, susceptibility of cervical spine to fracture during extension, and potentially limited mouth opening due to temporomandibular joint arthritis, which is seen in 10 per cent of patients 2 . The alternative technique, Spinal Anesthesia (SA), carries an unfavorably high failure rate. In a 10year review of anesthetic management of 13 patients with AS who underwent SA, the failure rate was reported to be $23.8 \% 3$. Syndesmophytes, thoracic kyphosis, loss of lumbar lordosis and loss of flexibility of the vertebral joints are potential contributing factors to the technical difficulty of SA4.

Here, we describe a case of preoperative three-dimensional computed tomography followed by real-time ultrasound-guided SA in a patient with severe AS and a high risk for general anaesthesia. To date, there is no documentation of the use of this combination of techniques for patients with difficult SA.

\section{Case Presentation}

A woman in her eighties was transferred to our hospital for further management of a left intertrochanteric femoral fracture, considering a potential need for cardiothoracic surgical support. She had severe AS, an asymptomatic retrosternal goitre, 
well-controlled hypertension, and New York. Heart Association Class II congestive heart failure. She was able to walk with a stick and care for herself prior to sustaining a fall at home.

\section{Clinical Findings}

The patient exhibited restricted neck movements on examination.

\section{Diagnostic Assessment}

Computed Tomography (CT) of the thorax (figure 1) a grossly enlarged multinodular thyroid gland with retrosternal extension abutting the brachiocephalic trunk at the T3 level. The trachea was deviated to the left side with focal narrowing due to displacement of the right thyroid lobe. The narrowest luminal thickness was approximately three millimeters, at the level of the clavicle. An irregular consolidation area was observed in the right lower lobe, in addition to bilateral pleural effusion, suggestive of infective changes.

Preoperative radiographs of the cervical (figure 2) and lumbrosacral spine (figure 3) showed fusion of facet joints with symmetrical syndesmophyte formation, giving the typical appearance of a "bamboo spine." CT of the lumbrosacral spine confirmed diffuse fusion of bilateral sacroiliac, facet, and costovertebral joints. Diffuse syndesmophyte formation and interspinous ligamental ossification were also noted. A threedimensional CT (3D-CT) reconstruction of the lumbrosacral spine (figure 4) showed unfused interlaminar spaces on the left side at the L2-3 level (figure 5a) and on the right side at the L3-4 level (figure 6a), measuring $11 \mathrm{~mm}$ (figure 5b) and 6.3 $\mathrm{mm}$ (figure $6 \mathrm{~b}$ ), respectively, in the longitudinal axis.

Following discussion with the patient and her family by a multidisciplinary team, a joint decision was made to proceed with surgical fixation of intertrochanteric femoral fracture under SA.

\section{THERAPEUTIC INTERVENTION}

Rigid bronchoscopy was contraindicated in the patient due to limited neck extension. Sternotomy and extracorporeal membrane oxygenator were offered as contingency plans if the pa- tient develop total airway obstruction. Standard monitoring was performed, and an arterial line was inserted. Ultrasoundguided fascia iliaca block with $20 \mathrm{ml}$ of $0.2 \%$ ropivacaine was performed for analgesia before positioning. Real-time US-guided SA was performed by two operators under aseptic conditions. Following identification of the transverse processes and interarticular joints of the lumbar spine in the paramedian sagittal view (figure 7 and 8), the paramedian sagittal oblique view was obtained on both sides. The posterior and anterior complexes were visible only at the L2-3 level on the left side (figure 9) and L3-4 level on the right side (figure 10), measuring $7.7 \mathrm{~mm}$ and $6.0 \mathrm{~mm}$, respectively, in the longitudinal axis. The curved probe of the US was held in place by one anesthetist, while the other advanced an $18 \mathrm{G}$ Tuohy needle after skin and subcutaneous infiltration with $2 \%$ lidocaine. By using an in-plane approach, the needle was followed under constant vision and directed towards the posterior complex (figure 11). Free flow of clear cerebrospinal fluid was observed upon withdrawal of the stylet and $2.6 \mathrm{ml} 0.5 \%$ levobupivacaine were injected intrathecally after negative aspiration.

\section{Outcome}

The effect of SA was confirmed by the loss of sensation to ice bilaterally at the T10 level. Proximal femoral nail antirotation was subsequently performed uneventfully and the patient remained haemodynamically stable throughout and after surgery. Discussion and Conclusion

This case was challenging for several reasons. Her limited range of neck movements posed a high degree of technical difficulty for tracheal intubation. Although this could be circumvented by using a fiberoptic technique or supraglottic airway device, the presence of a retrosternal goiter occupying the anterior mediastinum was a much greater concern, since it could cause total airway obstruction under general anaesthesia. Moreover, pneumonia had reduced her respiratory reserve, thus limiting the duration of intubation apnoea. In the event of total airway obstruction, the default option of rigid bronchoscopy as a rescue plan was contraindicated due to restricted neck movement.

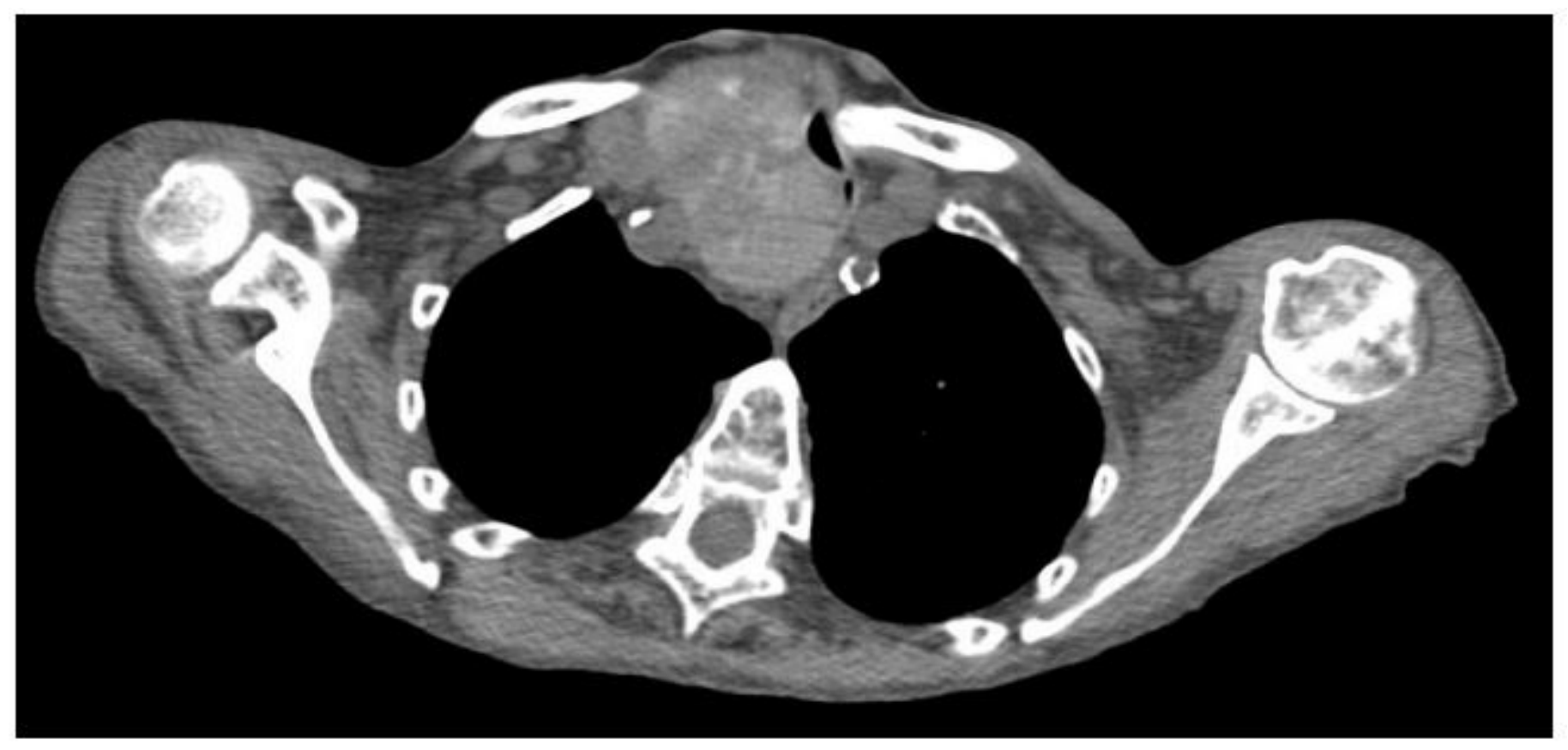

Figure 1 : CT thorax showing a grossly enlarged multinodular thyroid gland with retrosternal extension. The tracheais deviated to the left side with the narrowest diameter measuring about $3 \mathrm{~mm}$ at the level of the clavicle. 

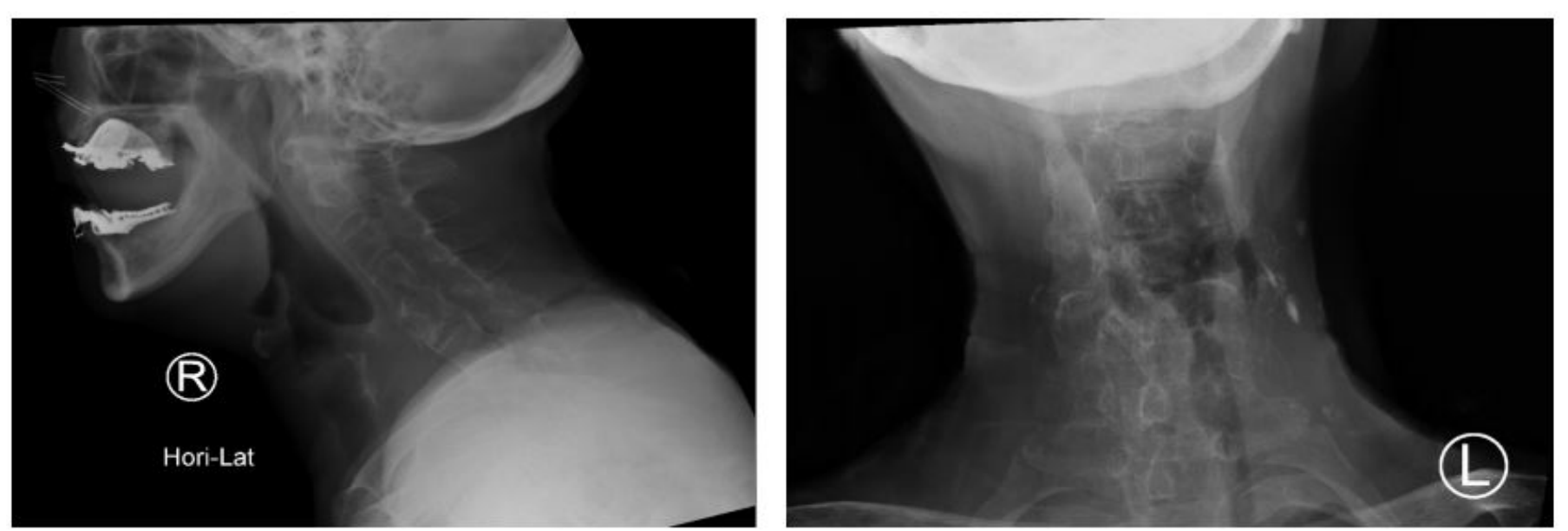

Figure 2: Radiograph of the cervical spine showing fusion of facet joints with symmetrical syndesmophyte formation.
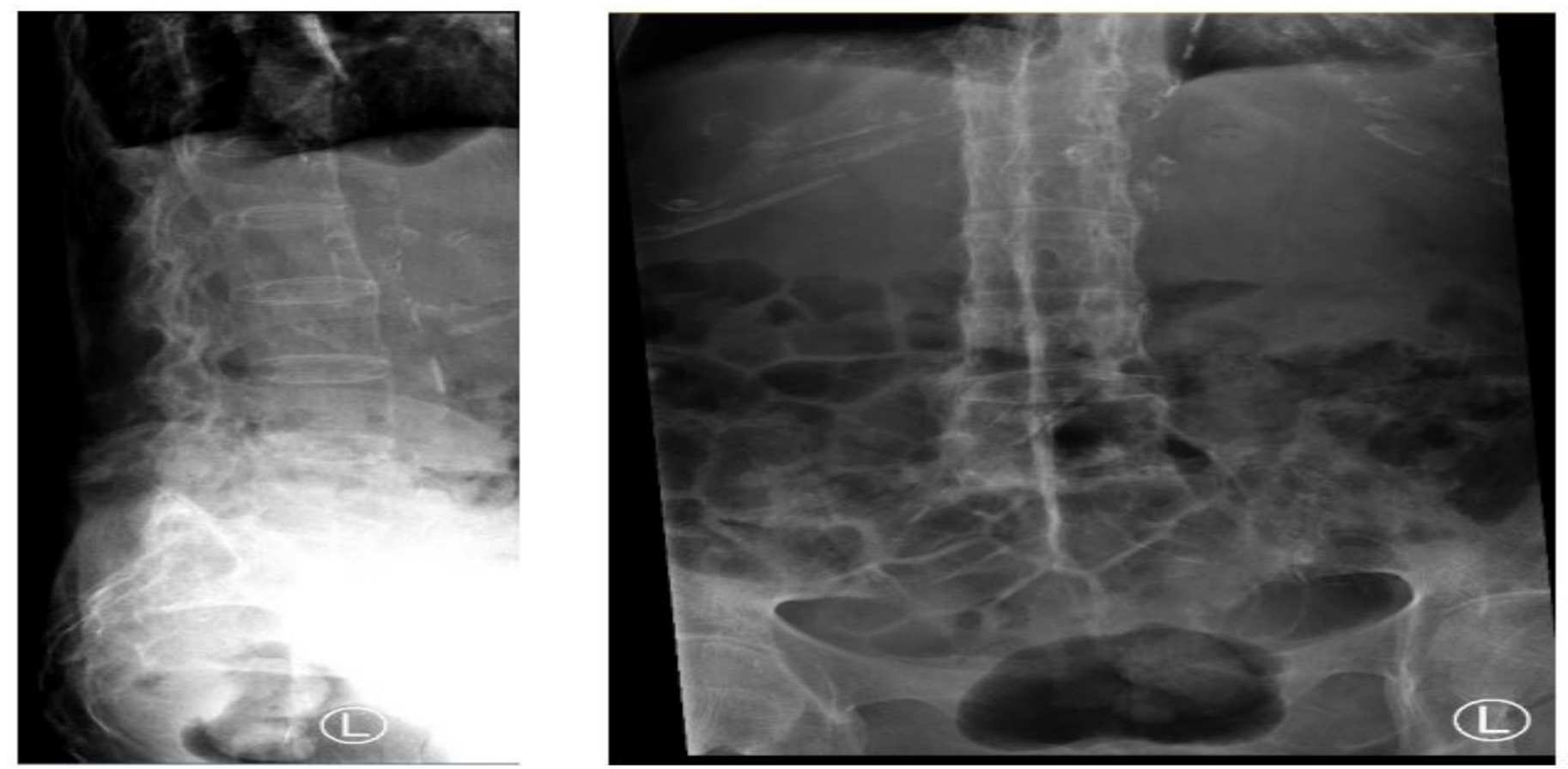

Figure 3 : Radiograph of the lumbrosacral spine showing typical radiological appearance of a "bamboo spine."

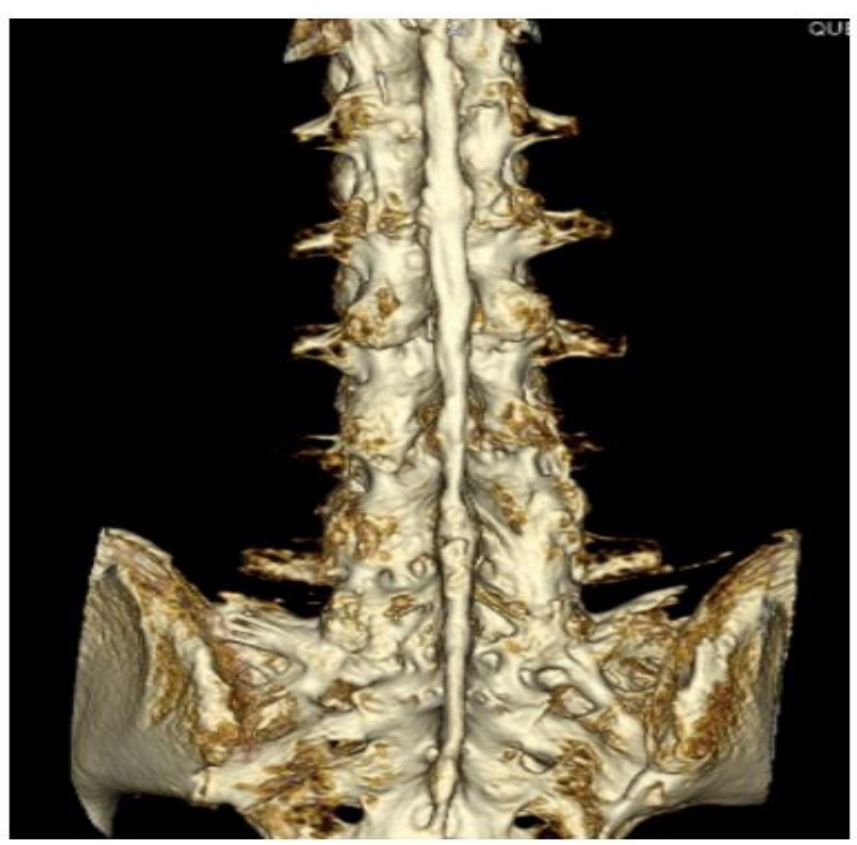

Figure 4: Three-dimensional CT reconstruction of the lubrosacral spine.
Therefore, emergency sternotomy or pre-emptive placement of extracorporeal membrane oxygenator were the only viable options for airway and ventilatory support. Therefore, general anaesthesia was deemed as a very high-risk procedure.

The hip and thigh are innervated by branches of the lumbrosacral plexus, namely, the femoral nerve, obturator nerve, and lateral femoral cutaneous nerve from the lumbar plexus, and the sciatic nerve, posterior cutaneous nerve of the thigh, and superior gluteal nerve from the sacral plexus. Performing regional anesthesia by blocking the plexus or all branches is technically challenging and entails a high risk of local anaesthetic systemic toxicity due to the high volume of local anaesthetic that would be required to allow adequate coverage. Furthermore, in this case, use of sedation as a supplement could lead to a reduction in muscle tone, resulting in total airway obstruction. Therefore, regional anaesthesia was not considered a safe option for the patient. It has been reported that asymptomatic patients with tracheal stenosis of more than $50 \%$ of the residual cross area, as in our patient, are considered to be at high risk of developing intraoperative airway problems 5 .

Pre-procedural imaging of the spine in this particular patient 

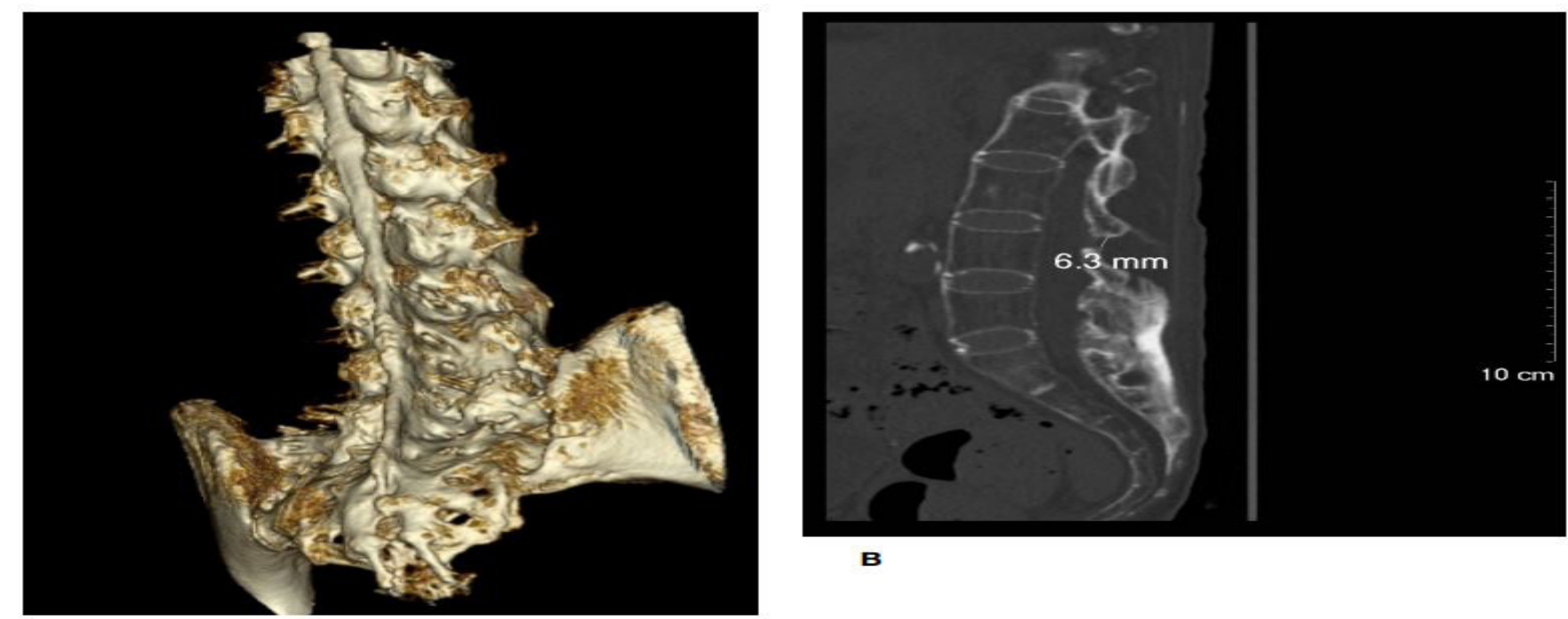

A

Figure 5: An unfused interlaminar space identified on the left side at the L2/3 level (figure 5a), measuring $11 \mathrm{~mm}$ in the longitudinal axis (figure $5 b$ ). The corresponding axial view is shown in figure $5 c$.
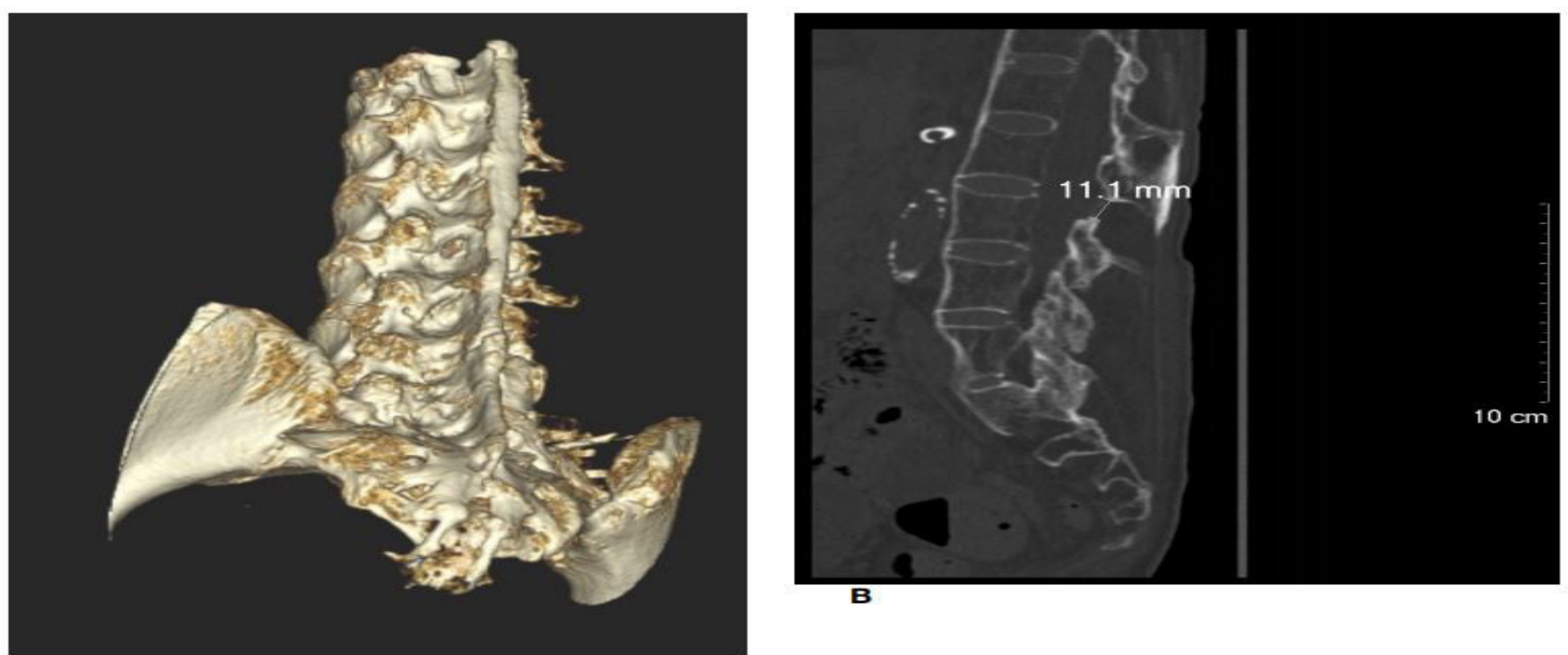

$\mathbf{B}$

A

Figure 6: Another unfused interlaminar space identified on the right side at the L3/4 level (figure 6a), measuring 6.3 mm in the longitudinal axis (figure 6b). The corresponding axial view is shown in figure $6 c$.

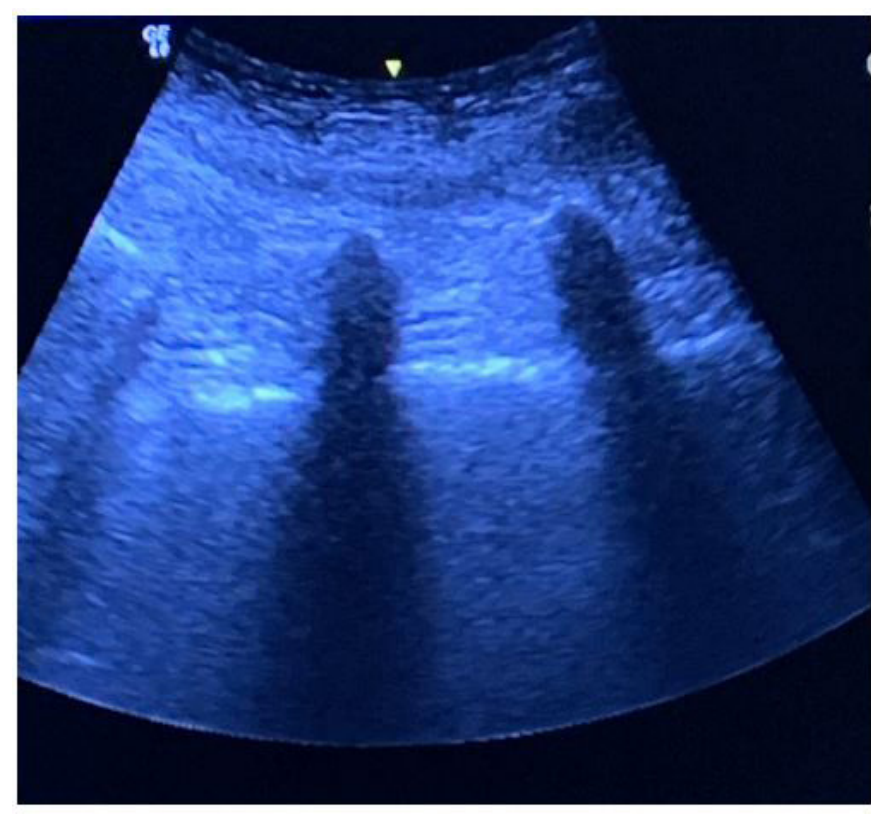

Figure 7: Ultrasound image of the transverse processes of the lumbar spine obtained from the paramedian sagittal view.

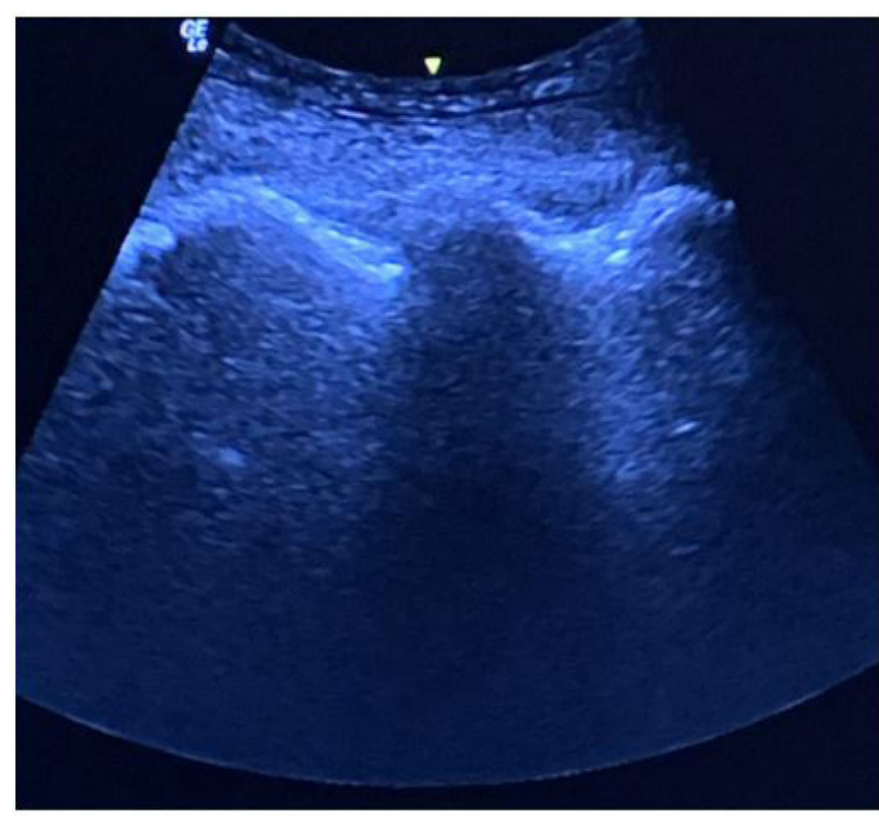

Figure 8: Ultrasound image of the interarticular joints of the lumbar spine obtained from the paramedian sagittal view. 


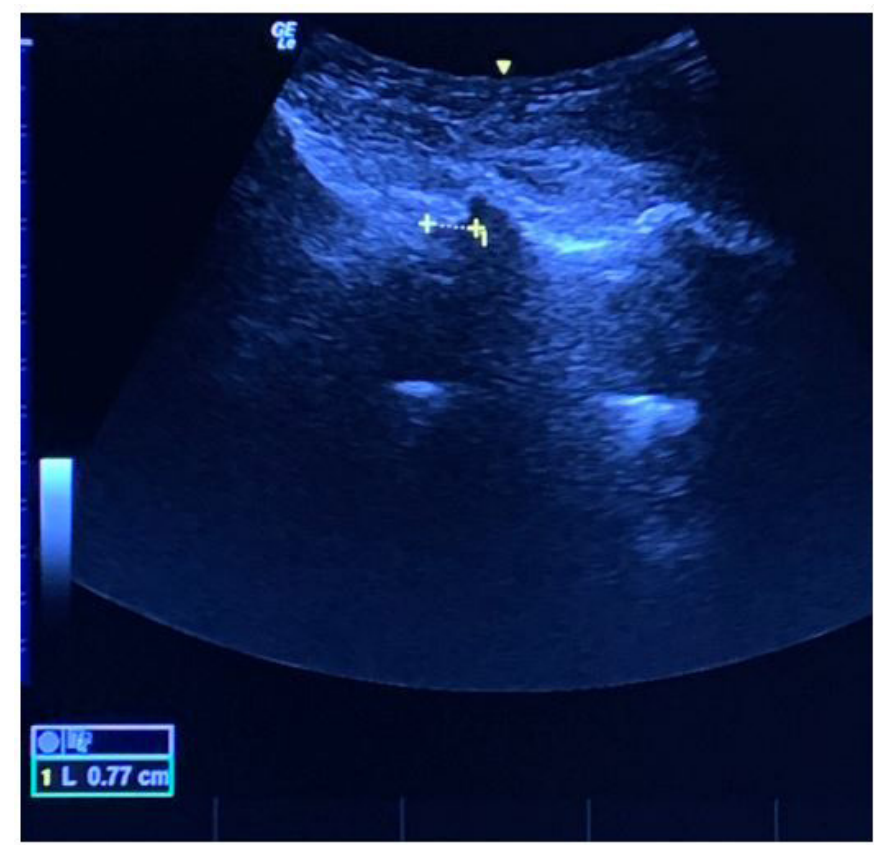

Figure 9: The posterior and anterior complexes visible only at the L2/3 level on the left side (figure 9a), measuring $7.7 \mathrm{~m}$ in the longitudinal axis (figure $9 \mathrm{~b}$ ).

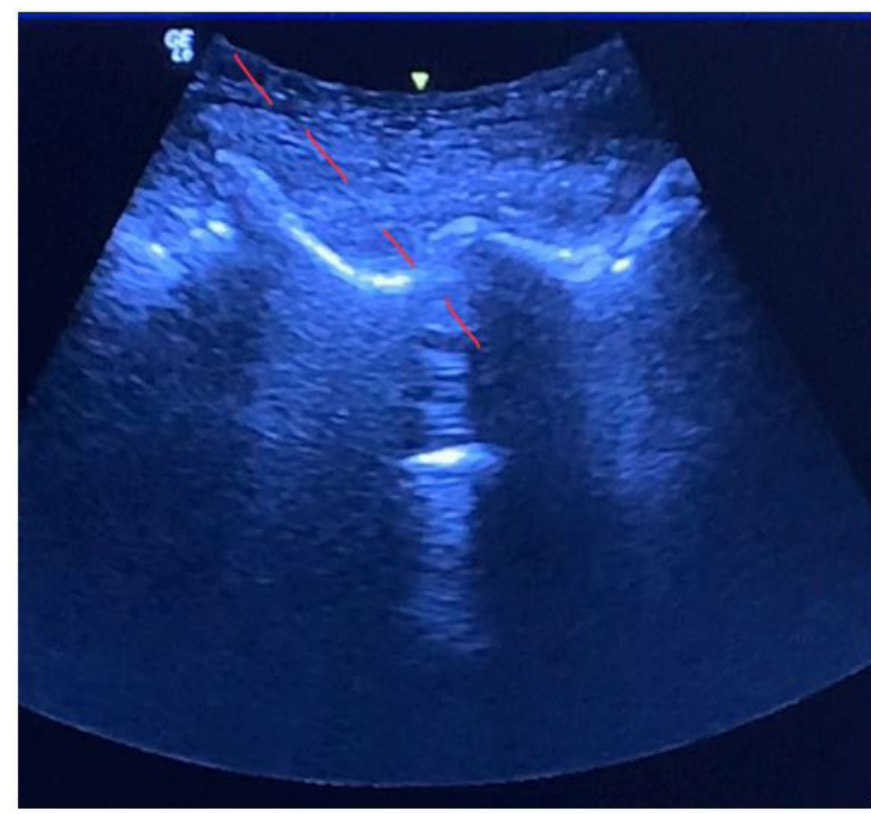

Figure 11: The needle is seen directed towards the posterior complex with an in-planeapproach.

group has been described in a few studies6,7,8 CT and MRI are more sensitive than plain radiographs for assessing the involvement of the spine8. Three-dimensional CT is best for demonstrating interspinous and interlaminar spaces, whereas MRI is best for demonstrating soft tissue inflammation6. Compared to US, 3D-CT is non-operator-dependent, thus providing an objective means of identifying unfused interlaminar spaces for a preoperative assessment regarding the feasibility of SA. Various SA techniques have also been explored, including USguided 8 and fluoroscopy-guided9,10 Taylor's approach11 and mini-laminectomy 12. Both US and fluoroscopy are minimally invasive and are familiar techniques in our institute. However, the latter requires the patient to be laid in a prone position, which can be distressing and potentially hazardous in a patient with a fractured hip and an obstructive retrosternal goitre.

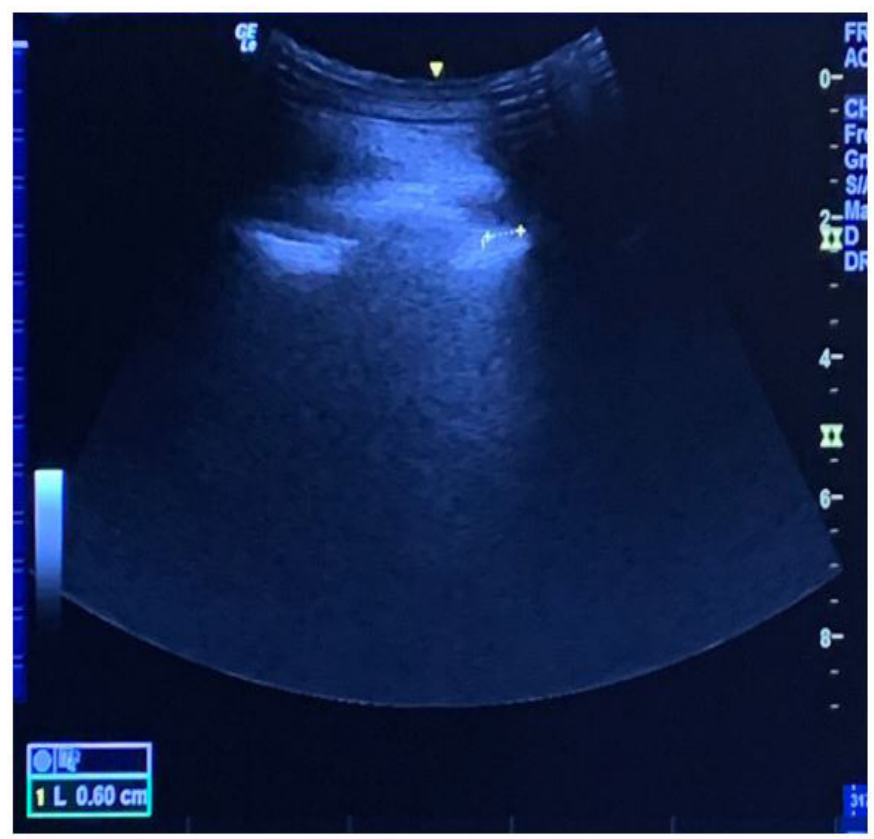

Figure 10: The posterior and anterior complexes visible only at L3/4 level on the right side (figure 10a), measuring $6.0 \mathrm{~mm}$ in the longitudinal axis (figure 10b).

Moreover, fluoroscopy exposes the patient and operators to radiation, thereby making fluoroscopy-guided SA a less favorable choice.

The potential of US in predicting the ease of performing neuraxial anesthesia has been evaluated in two cohort studies 13,14. These were based on the premise that the ability to visualise the vertebral canal corresponds to the size of the interlaminar space, thus reflecting the ease with which it may be penetrated. However, the angle of the US scan in attaining the most optimal view and, therefore, the path of needle advancement may not be accurately replicated during the actual procedure. This may subsequently lead to failure, especially in patients with challenging anatomies. Real-time US-guided SA overcomes this limitation by allowing manipulation of the needle under direct vision. This advantage is illustrated in our case by the fact that redirection of the needle was not required, and SA was conducted in a single attempt once the optimal view was acquired.

Although US has been shown to allow identification of interlaminar spaces, epidural/intrathecal spaces, and adjacent structures 15 , its accuracy is affected by multiple factors, including the quality of US equipment, angle of US beam penetration, patient positioning, and operator technique. These factors may account for the discrepancy in measured sizes of the interlaminar spaces by US and 3D-CT. Another caveat of this technique is the technical difficulty in maintaining the desired view on US while manipulating the needle. We opted to have two operators to perform each of these tasks; however, several single-operator techniques have been described in other studies. Visualising the needle on US can be challenging because of the steep angle of needle advancement and the low-frequency transducer that is typically used in US. Ideally, echogenic spinal needles should be used; however, since they were not available in our institute, the $18 \mathrm{G}$ Tuohy needle was chosen for better visibility rather than non-echogenic spinal needles. 
In conclusion 3D-CT provides an objective way of identifying unfused interlaminar spaces to assess the feasibility of a SA while real-time US-guided SA offers the advantage of allowing manipulation of needle under direct vision without exposure to radiation.

\section{Patient Perspective}

My family and I understood the implication of having an obstructive mass in the chest and. On the other hand, the fracture was so painful that it impacted my independence and quality of life. We had therefore agreed to proceed with the surgery despite the high risk my anaesthetic would entail.

In the end, we are thankful to the thorough explanation and planning done by the team. We are grateful that the surgery and the anaesthetic went well.

(Translated from Cantonese)

\section{Declaration}

Ethics approval and consent to participate: Not applicable for case reporting.

\section{Consent for publication:}

The patient's family has provided written consent to publish this case report.

\section{Availability of data and materials:}

Not applicable to this article as no datasets were generated or analysed in this report.

\section{Competing Interest:}

None

Funding:

None

\section{Authors' contribution}

DL: This author helped with the conception and revision of the work. He approved the version to be published and agreed to be accountable for all aspects of the work in ensuring that questions related to the accuracy or integrity of any part of the work are appropriately investigated and resolved.

JC: This author helped with the design and drafting of the work. He approved the version to be published and agreed to be accountable for all aspects of the work in ensuring that questions related to the accuracy or integrity of any part of the work are appropriately investigated and resolved.

HM: This author helped with the design and drafting of the work. He approved the version to be published and agreed to be accountable for all aspects of the work in ensuring that questions related to the accuracy or integrity of any part of the work are appropriately investigated and resolved.
YFC: This author helped with the design and drafting of the work. He approved the version to be published and agreed to be accountable for all aspects of the work in ensuring that questions related to the accuracy or integrity of any part of the work are appropriately investigated and resolved.

TC: This author helped with the conception and revision of the work. He approved the version to be published and agreed to be accountable for all aspects of the work in ensuring that questions related to the accuracy or integrity of any part of the work are appropriately investigated and resolved.

All authors have read and approved the manuscript.

\section{Acknowledgement:}

none.

\section{References}

1. Woodward LF, Kam PCA. Ankylosing spondylitis: recent developments and anaesthetic implications. Anaesthesia 2009; 64: 540-548.

2. Zhao J, Huang $\mathrm{C}$, Huang $\mathrm{H}$, et al. Prevalence of ankylosing spondylitis in a Chinese population: a systematic review and meta-analysis. Rheumatol Int 2020; 40: 859-872.

3. Schelew BL, Vaghadia H. Ankylosing spondylitis and neuraxial anaesthesia--A 10 year. Can J Anaesth, Volume 43: 65-68. Can j anesth 1996; 65-68.

4. Ozturk I, Ozkan D, Ergil J. A systematic review of neuraxial anesthesia in patients with Ankylosing spondylitis. EJMO. 2017; 1(3): 119-123.

5. Ng A, Bennett J, Bromley P, Davies P, Morland B. "Anaesthetic outcome and predictive risk factors in children with mediastinal tumours," Pediatric Blood and Cancer, 2007; 48(2): 160-64.

6. Soh E, Karmakar MK. Assessment of the spine with CT and MRI prior tointerspinous/interlaminar spinal procedures: a pictorial review. BJR, 2013; 86(1026): 20130066.

7. Lacout A, Rousselin B, Pelage J-P. CT and MRI of spine and sacroiliac involvement inspondyloarthropathy. AJR Am j roentgenol 2008; 191: 1016-1023.

8. Chin KJ, Chan V. Ultrasonography as a preoperative assessment tool: Predicting the Feasibility of Central Neuraxial Blockade, Anesth analg 2010; 252-253.

9. Channabasappa SM, Dharmappa S, Pandurangi R. Fluoroscopy guided transforaminalepidural anesthesia in ankylosing spondylitis. Saudi j anaesth 2016; 101-103.

10. Gupta M, Gupta P. Fluoroscopic-guided paramedian approach to subarachnoid block inpatients with ankylosing spondylitis: A case series. Indian j anaesth 2018; 142-144.

11. Srivastava A, Arora A, Gupta D, Asthana V. Ultrasound-guided Taylor's approach inankylosing spondylitis. Anesth Essays res 2018; 12: 761-764.

12. Leung KH, Chiu KY, Wong YW, Lawmin JC. Case report: Spinal anesthesia by mini-laminotomy for a patient with ankylosing spondylitis who was difficult to anesthetize. Clin, Volume 468: 3415-3418 Orthop relat Res 2010; 468: 3415-3418. 
